\title{
Proportional Fairness in 802.11 Wireless LANs
}

\author{
A.Checco, D.J.Leith \\ Hamilton Institute, NUI Maynooth
}

\begin{abstract}
We provide the first rigorous analysis of proportional fairness in 802.11 WLANs. This analysis corrects prior approximate studies. We show that there exists a unique proportional fair rate allocation and completely characterise the allocation in terms of a new air-time quantity, the total air-time.
\end{abstract}

\section{INTRODUCTION}

Proportional fairness has been the subject of considerable attention in the literature on multi-rate 802.11 WLANs since it can be used to address the performance "anomaly" [3] in a principled manner and is closely related to so-called time-based fairness $[4,11]$. Well established utility fairness techniques from wired networks cannot, however, be directly applied to random access CSMA/CA wireless networks due to the presence of collision losses and the coupling of station transmissions via carrier sense.

For Aloha wireless networks, proportional fairness is rigorously analysed in $[5,7,12]$. In $[5,12]$ it is established that there exists a unique proportional fair rate allocation and local message-passing algorithms that converge to this allocation are proposed. In [7] an alternative distributed algorithm based on back-pressure is studied. However, there are no corresponding rigorous results for 802.11 WLANs and the literature is confined to approximate approaches $[1,2,4,8,10]$. The work in $[4,8]$ neglects collision losses while [1] neglects collisions involving more than two stations. In [10] an algorithm based on contention window tuning is proposed and in [2] an algorithm using MTU tuning is proposed. Notwithstanding this work, since the 802.11 rate region is non-convex basic issues such as the existence and uniqueness of proportional fair rate allocations remain open.

It is notable that these approximate approaches in the literature share in common the idea that proportional fairness is related to some form of air-time fairness. In this paper we provide the first rigorous analysis of proportional fairness in 802.11 WLANs. For a WLAN (i.e. a single wireless hop) with no hidden terminals or noise losses we show that there exists a unique proportional fair rate allocation and completely characterise the allocation in terms of an air-time quantity. Importantly, we find that the correct air-time quantity, the flow total air-time, differs from the quantities previously considered in the literature. Our analysis is general enough to encompass both per station fairness and per flow fairness, and does not assume symmetric network load (stations may have different numbers of flows and flows may have finite offered load, leading to stations being unsaturated). Our work builds on the recent observation that the 802.11 rate region is log-convex [6].

This material is based upon works supported by Science Foundation Ireland under Grant No. 07/IN.1/1901.

\section{NeTwORK MODEL}

1) Station Throughput: We consider an 802.11 WLAN with $n$ stations, $n \geq 2$. Let $\tau_{i}$ denote the probability that station $i$ attempts a transmission. The throughput of station $i$ is [6]:

$$
S_{i}(\tau)=\frac{P_{\text {succ }, i} D_{i}}{\sigma P_{\text {idle }}+T_{c}\left(1-P_{\text {idle }}\right)}
$$

where $P_{i d l e}=\prod_{k=1}^{n}\left(1-\tau_{k}\right), P_{\text {succ }, i}=\tau_{i} \prod_{k=1, k \neq i}^{n}\left(1-\tau_{k}\right)$, $\tau=\left[\begin{array}{lll}\tau_{1} & \ldots & \tau_{n}\end{array}\right]^{T}, \sigma$ is the PHY idle slot duration, $T_{c}$ the mean duration of a transmission, $D_{i}$ the mean number of bits sent by station $i$ in a successful transmission. This model is from [6], with the mean durations of successful and colliding transmissions taken equal i.e. without TXOP packet bursting.

The WLAN rate region is the set $\mathcal{R}$ of achievable throughput vectors $S(\tau)=\left[\begin{array}{lll}S_{1} & \ldots & S_{n}\end{array}\right]^{T}$ as the vector $\tau$ of attempt probabilities ranges over domain $[0,1]^{n}$. We also define the $\log$-transformed rate region $\tilde{\mathcal{R}}$ as the set of achievable vectors $\tilde{S}(\tau)=\left[\begin{array}{lll}\tilde{S}_{1} & \ldots & \tilde{S}_{n}\end{array}\right]^{T}, \tilde{S}_{i}=\log S_{i}$.

It will prove useful to work in terms of $x_{i}=\tau_{i} /\left(1-\tau_{i}\right)$ rather than $\tau_{i}$; observe that $x_{i} \in[0, \infty)$ for $\tau_{i} \in[0,1)$. We have that $P_{\text {idle }}=1 / \prod_{k=1}^{n}\left(1+x_{k}\right), P_{\text {succ }, i}=x_{i} P_{\text {idle }}$ and

$$
S_{i}(x)=\frac{x_{i}}{X(x)} \frac{D_{i}}{T_{c}}
$$

where $X(x)=a+\prod_{k=1}^{n}\left(1+x_{k}\right)-1$ with $a=\sigma / T_{c}$.

2) Flows: We assign the packets transmitted by each station to "flows". Let $\mathcal{P}_{i}$ be the set of flows carried by station $i$ and $\mathcal{P}=\cup_{i=1}^{n} \mathcal{P}_{i}$ the set of flows in the WLAN. How we choose to define a flow is essentially a design decision, subject only to the constraint that $\sum_{p \in \mathcal{P}_{i}} s(p)=S_{i}(x), s(p)$ the rate of flow $p$. For example, we might define a flow to consist of packets with the same source-destination address/port number quadruple. Alternatively, per-station fairness is subsumed within our formulation by defining all packets transmitted by the same station to be a flow.

3) Air-time: We clarify air-time, since in the literature various non-equivalent definitions are used.

Definition 1 (Transmission Duration). The time $T_{c}$ taken to transmit a frame; e.g., used in [2].

Definition 2 (Successful Station Air-time). The fraction of time spent by a station on successful transmissions; e.g., used in [1]. For station $i$, this is given by $T_{\text {succ }, i}=x_{i} / X(x)$.

Definition 3 (Flow Total Air-time). The fraction of time $T(p)$ used for transmissions by flow $p$, including both successful transmissions and collisions. We also define the station total air-time $T_{i}=\sum_{p \in \mathcal{P}_{i}} T(p)$ and note that

$$
T_{i}=\frac{\tau_{i} P_{\text {coll }, i} T_{c}+\tau_{i}\left(1-P_{\text {coll }, i}\right) T_{c}}{\sigma P_{\text {idle }}+T_{c}\left(1-P_{\text {idle }}\right)}=\frac{x_{i}}{X(x)}\left(1+\frac{P_{\text {coll }, i}}{1-P_{\text {coll }, i}}\right)
$$


where $P_{\text {coll }, i}=1-\frac{\prod_{k=1}^{n}\left(1-\tau_{k}\right)}{1-\tau_{i}}=1-\frac{1+x_{i}}{\prod_{k=1}^{n}\left(1+x_{k}\right)}$ is the collision probability experienced by station $i$.

\section{Proportional FAIR RATE Allocation}

Assume that the WLAN offered load is unconstrained. This is similar to a saturated station (i.e. stations always have a packet to send) assumption, which we will later relax. To determine the proportional throughput allocation we need to solve the following utility optimisation problem:

$$
\max _{x, s} \sum_{p \in \mathcal{P}} \log s(p) \text { s.t. } \sum_{p \in \mathcal{P}_{i}} s(p) \leq \frac{x_{i}}{X(x)} \frac{D_{i}}{T_{c}}, x_{i} \geq 0, i=1, \ldots, n
$$

The constraints ensure that the aggregate flow throughput at station $i$ is feasible and so lies within the WLAN rate region. Since the WLAN rate region $\mathcal{R}$ is non-convex, the optimisation problem is non-convex. Fortunately, it has recently been shown that the log-transformed rate region $\tilde{\mathcal{R}}$ is, however, convex [6]. Changing variables to $\tilde{s}(p)=\log s(p)$, the optimisation can therefore be transformed into convex form:

Problem 1 (Proportional Fairness).

$$
\max _{x, s} \sum_{p \in \mathcal{P}} \tilde{s}(p) \text { s.t. } \log \sum_{p \in \mathcal{P}_{i}} e^{\tilde{s}(p)} \leq \log \frac{x_{i} D_{i}}{X(x) T_{c}}, x_{i} \geq 0, i=1, . ., n
$$

In addition to the constraints now being convex, there also exists a strictly feasible point and so the Slater conditions [9] are satisfied. Hence, strong duality holds and the KKT conditions [9] are necessary and sufficient conditions for global optimality. However, since the objective function is not strictly concave (it is linear in $\tilde{s}(p)$ ), extra work is needed to establish that the optimisation has a unique solution. We will make use of the following Lemma:

Lemma 1. The log-transformed rate region $\tilde{R}$ is strictly convex.

Proof: Let $\partial \tilde{\mathcal{R}}$ denote the boundary of set $\tilde{\mathcal{R}}$. Since we already know that $\tilde{\mathcal{R}}$ is convex, to establish strict convexity it is sufficient to show that the tangent hyperplanes to any two boundary points $\tilde{S}\left(x^{1}\right), \tilde{S}\left(x^{2}\right) \in \partial \tilde{\mathcal{R}}$ are different whenever $x^{1} \neq x^{2}$. Now $\tilde{S}_{i}(x)=\log \frac{x_{i}}{X(x)} \frac{D_{i}}{T_{c}}$ and

$$
\frac{\partial \tilde{S}_{i}}{\partial x_{j}}=\frac{\delta_{i j}}{x_{i}}-\frac{1}{X} \prod_{\substack{k \neq j \\ k=1}}^{n}\left(1+x_{k}\right)
$$

where $\delta_{i j}=1$ when $i=j$ and 0 otherwise. The normal vector $b(x)$ to the tangent hyperplane at point $\tilde{S}(x) \in \partial \tilde{\mathcal{R}}$ solves

$$
\sum_{i=1}^{n} b_{i}(x) \frac{\partial \tilde{S}_{i}(x)}{\partial x_{j}}=0 \forall j=1, \ldots, n .
$$

It can be verified that $b_{j}(x)=\frac{x_{j}}{1+x_{j}}$ is one such normal vector. Hence, for any two points $\tilde{S}\left(x^{1}\right), \tilde{S}\left(x^{2}\right)$ on the rate region boundary with $x^{1} \neq x^{2}$, the corresponding tangent hyperplanes are different, as required.

We are now in a position to state the first of our main results.

Theorem 1. There exists a unique proportional fair rate allocation, the solution to Problem 1, which: (i) assigns equal flow total air-times $(T(p)=T(q) \forall p, q \in \mathcal{P})$, (ii) the flow total air-times sum to unity $\left(\sum_{p \in \mathcal{P}} T(p)=1\right)$ and (iii) lies on the boundary of the WLAN rate region. Note that since the flow air-time usage overlaps due to collisions, (ii) does not imply that the channel idle probability $P_{\text {idle }}=0$.

Proof: We proceed by making use of strong duality. The Lagrangian is

$$
L(x, s, \lambda)=\sum_{p \in \mathcal{P}} \tilde{s}(p)+\sum_{i=1}^{n} \lambda_{i}\left(\log \frac{x_{i} D_{i}}{X T_{c}}-\log \sum_{p \in \mathcal{P}_{i}} e^{\tilde{s}(p)}\right)
$$

The main KKT conditions are

$$
\begin{array}{lrl}
1-\lambda_{i} \frac{e^{\tilde{s}(p)}}{\sum_{q \in \mathcal{P}_{i}} e^{\tilde{s}(q)}}=0 & \forall p \in \mathcal{P}, i=r(p) \\
\frac{\lambda_{i}}{x_{i}}-\frac{1}{X} \frac{\partial X}{\partial x_{i}} \sum_{j=1}^{n} \lambda_{j}=0, & i=1, \ldots n
\end{array}
$$

From the first KKT condition (2), $s(p)=\sum_{q \in \mathcal{P}_{i}} s(q) / \lambda_{i} \forall p \in$ $\mathcal{P}_{i}$ and so all flows carried by the same station are allocated the same throughput. Summing (2) over flows $p \in \mathcal{P}_{i}$ carried by station $i$ yields

$$
\lambda_{i}=n_{i}
$$

where $n_{i}=\left|\mathcal{P}_{i}\right|$ is the number of flows carried by station $i$. It follows from complementary slackness that since $\lambda_{i}>0$ the station throughput constraint in Problem 1 is tight. Thus station throughput $S_{i}(x)=n_{i} s(p)$ and $\tilde{S}_{i}(x)=\log n_{i} s(p)$. The contribution to the optimisation objective function in Problem 1 by station $i$ is $n_{i} \log s(p)=n_{i} \tilde{S}_{i}-n_{i} \log n_{i}$. Hence, the level sets form hyperplanes in rate region $\tilde{R}$. Any optimal solution $\tilde{S}^{*}$ must lie on the boundary of $\tilde{R}$, else the flow throughputs could be increased and the objective improved. Since $\tilde{R}$ is strictly convex by Lemma 1, each boundary point has a unique supporting hyperplane and so the optimum of Problem 1 is unique, as claimed.

Turning now to the second KKT condition, rearranging (3) and using (4) yields $T_{i}=\frac{\lambda_{i}}{\sum_{j=1}^{n} \lambda_{j}}$, where $T_{i}$ is the total airtime used by station $i$. The total air-time used by each flow $p \in \mathcal{P}_{i}$ carried by station $i$ is

$$
T(p)=\frac{T_{i}}{n_{i}}=\frac{1}{\sum_{j=1}^{n} \lambda_{j}}
$$

establishing the property (i) as claimed. Property (ii) follows from the fact that $\sum_{j=1}^{n} \lambda_{j}=\sum_{j=1}^{n}\left|\mathcal{P}_{j}\right|=|\mathcal{P}|$.

\section{FINITE-LOAD}

The foregoing analysis can be generalised to situations with finite offered-load. Problem 1 is then augmented with the additional (convex) constraint

$$
\tilde{s}(p) \leq \log \bar{s}(p), \quad \forall p \in \mathcal{P}
$$

where $\bar{s}(p)>0$ is the offered load of flow $p$. Let $\mathcal{S}=$ $\{r \in \mathcal{P}: s(r)=\bar{s}(r)\}$ denote the set of offered-load constrained flows and $\mathcal{U}=\mathcal{P} \backslash \mathcal{S}$ the set of network constrained flows. We have the following intuitive extension of Theorem 1 demonstrating that the air-time left unused by the offeredload constrained flows is simply reallocated equally amongst the network constrained flows: 
Theorem 2. The proportional fair rate allocation with offeredload constraint (5): (i) assigns equal total air-time to all network constrained flows $(T(p)=T(q) \forall p, q \in \mathcal{U})$, (ii) the flow total air-times sum to unity $\left(\sum_{p \in \mathcal{P}} T(p)=1\right)$ and (iii) is on the boundary of its rate region.

Proof: Strong duality also holds for the augmented problem. The Lagrangrian is

$$
\begin{aligned}
L(x, s, \lambda, \theta)=\sum_{p \in \mathcal{P}} \tilde{s}(p) & +\sum_{i=1}^{n} \lambda_{i}\left(\log \frac{x_{i} D_{i}}{X(x) T_{c}}-\log \sum_{p \in \mathcal{P}_{i}} e^{\tilde{s}(p)}\right) \\
+ & \sum_{p \in \mathcal{P}} \theta_{p}(\overline{\tilde{s}}(p)-\tilde{s}(p))
\end{aligned}
$$

with multiplier $\theta_{p} \geq 0 \forall p \in \mathcal{P}$. The second KKT condition (3) is unchanged but the first KKT condition becomes

$$
1-\lambda_{i} \frac{e^{\tilde{s}(p)}}{\sum_{q \in \mathcal{P}_{i}} e^{\tilde{s}(q)}}-\theta_{p}=0 \quad \forall p \in \mathcal{P}, i=r(p)
$$

Let $\mathcal{S}_{i}=\mathcal{P}_{i} \cap \mathcal{S}$ be the set of offered-load constrained flows carried by station $i$ and $\mathcal{U}_{i}=\mathcal{P}_{i} \backslash \mathcal{S}_{i}$ the set of network constrained flows. For a network constrained flow $p \in \mathcal{U}_{i}$ multiplier $\theta_{p}=0$ by complementary slackness. Consider a station $i$ with at least one network constrained flow (if there are no network constrained flows, the throughput allocation is trivial). By (6), $s(p)=\sum_{q \in \mathcal{P}_{i}} s(q) / \lambda_{i} \forall p \in \mathcal{U}_{i}$ (so all network constrained flows on station $i$ have the same throughput) and

$$
s(r)=\bar{s}(r)=\left(1-\theta_{r}\right) s(p) \quad \forall r \in \mathcal{S}_{i}, p \in \mathcal{U}_{i}
$$

The multiplier $\theta_{r}$ is equal to the relative throughput difference between offered-load constrained flow $r$ and a network constrained flow. Combining these observations we have that

$$
\lambda_{i}=\sum_{p \in \mathcal{U}_{i}} 1+\sum_{r \in \mathcal{S}_{i}}\left(1-\theta_{r}\right)
$$

Now from the second KKT condition, $T_{i}=\frac{\lambda_{i}}{\sum_{j=1}^{n} \lambda_{j}}$. Taking this together with (7), the total air-time allocated to flow $p \in \mathcal{P}$ is

$$
T(p)=\left\{\begin{array}{lc}
\frac{1}{\sum_{j=1}^{n} \lambda_{j}} & p \in \mathcal{U}=\mathcal{P} \backslash \mathcal{S} \\
\frac{1-\theta_{p}}{\sum_{j=1}^{n} \lambda_{j}} & p \in \mathcal{S}
\end{array}\right.
$$

That is, every network constrained flow $p \in \mathcal{U}$ is allocated the same total air-time as claimed. Property (ii) follows from inspection of $\sum_{j=1}^{n} \lambda_{j}$. Operation at the rate region boundary is necessary as otherwise the flow throughputs could be increased and so the objective improved.

\section{EXAMPLE}

Consider a WLAN with 10 stations and 24 UDP flows. The WLAN uses $802.11 \mathrm{~g}$ MAC/PHY values ( $9 \mu \mathrm{s}$ slot time, short preamble, $6 \mathrm{Mbps}$ PHY rate). The first three stations carry 2,5 and 10 flows respectively. The remaining 7 stations carry one flow each. The offered load is not constrained, so Theorem 1 applies. Figure 1 shows the flow total airtimes and flow success air-times for the proportional fair rate allocation. It can be seen that the proportional fair allocation assigns equal flow total air-times but that that success air-times

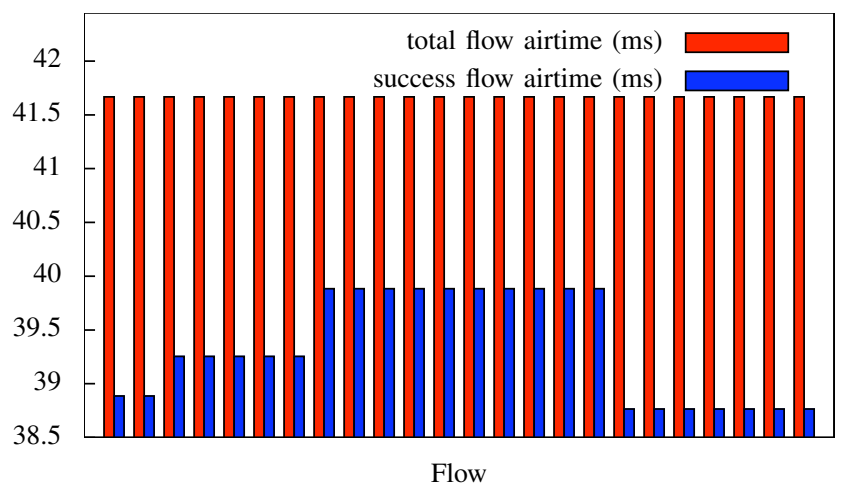

Fig. 1. Flow air-times of the proportional fair rate allocation in a $802.11 \mathrm{~g}$ WLAN with 24 UDP flows and 10 stations.

are unequal. In general, equalising flow total air-times is not equivalent to equalising flow success air-times whenever there is asymmetry in the network load (e.g. when stations do not all carry an identical number of flows) since this leads to stations experiencing different collision probabilities.

\section{CONCLUSIONS}

We provide the first rigorous analysis of proportional fairness in 802.11 WLANs. We show that a unique proportional fair rate allocation exists and, correcting previous studies, that this allocation assigns equal total air-time to flows. Total airtime is the air-time spent on both colliding and successful transmissions and differs from other air-time quantities proposed heuristically in the literature.

\section{REFERENCES}

[1] A Banchs, P Serrano, and H Oliver. Proportional Fair Throughput Allocation in Multirate IEEE 802.11e Wireless LANs. Wireless Networks, 13(5):649-662, 2006.

[2] J Dunn, M Neufeld, A Sheth, D Grunwald, and J Bennett. A Practical Cross-Layer Mechanism For Fairness in 802.11 Networks. Mobile Networks \& Appl., 11(1):37-45, 2005.

[3] M. Heusse, F. Rousseau, G. Berger-Sabbatel, and A. Duda. Performance Anomaly of 802.11b. Proc. INFOCOM 2003, pp 836-843, 2003.

[4] L Bin Jiang and S C Liew. Proportional Fairness in Wireless LANs and Ad hoc Networks. IEEE WCNC, 2005, pages 1551$1556,2005$.

[5] K. Kar, S Sarkar, and L Tassiulas. Achieving Proportional Fairness Using Local Information in Aloha Networks. IEEE Trans Aut Control, 9:1858-1863, 2004.

[6] D. J. Leith, V. Subramanian, and K. Duffy. Log-convexity of Rate region in $802.11 \mathrm{e}$ WLANs. IEEE Comms Letters, 14(1):57-59, 2010.

[7] J. Liu, A. L. Stolyar, M. Chiang, and V. Poor. Queue BackPressure Random Access in Multihop Wireless Networks: Optimality \& Stability. IEEE Trans Inf. Th., 55:4087-4098, 2009.

[8] T Nandagopal, T Kim, X Gao, and V Bharghavan. Achieving MAC Layer Fairness in Wireless Packet Networks. Proc. MobiCom '00, pp 87-98, 2000.

[9] R.T. Rockafellar. Convex analysis. Princeton Univ. Press. 1997.

[10] VA Siris and G Stamatakis. Optimal CWmin Selection for Achieving Proportional Fairness in Multi-rate 802.11e WLANs. Proc. WinTECH, pp. 41-48, 2006.

[11] G. Tan and J. Guttag. Time-based Fairness Improves Performance in Multi-rate WLANs. In Proc. USENIX, 2004.

[12] X Wang and K Kar. Cross-layer Rate Control for End-toend Proportional Fairness in Wireless Networks with Random Access. Proc MobiHoc, 2005. 